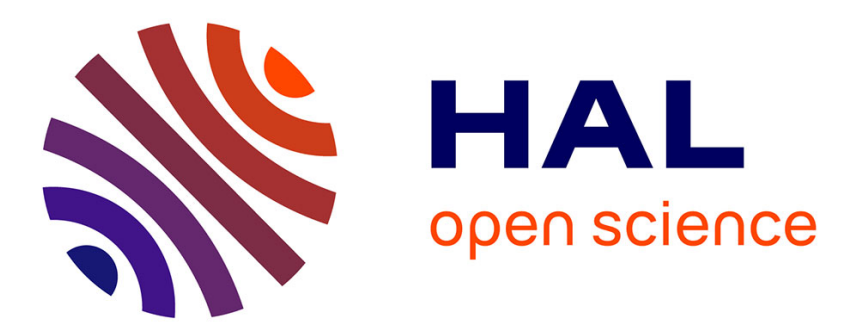

\title{
Les échelles d'équivalence complètes : une estimation intégrant les dimensions monétaire et temporelle des dépenses des ménages
}

François Gardes, Imen Sayadi, Christophe Starzec

\section{- To cite this version:}

François Gardes, Imen Sayadi, Christophe Starzec. Les échelles d'équivalence complètes : une estimation intégrant les dimensions monétaire et temporelle des dépenses des ménages. Revue d'Economie Politique, 2015, 125 (3), pp.393-414. 10.3917/redp.253.0393 . hal-01307097

\section{HAL Id: hal-01307097 https://hal.science/hal-01307097}

Submitted on 27 Apr 2016

HAL is a multi-disciplinary open access archive for the deposit and dissemination of scientific research documents, whether they are published or not. The documents may come from teaching and research institutions in France or abroad, or from public or private research centers.
L'archive ouverte pluridisciplinaire HAL, est destinée au dépôt et à la diffusion de documents scientifiques de niveau recherche, publiés ou non, émanant des établissements d'enseignement et de recherche français ou étrangers, des laboratoires publics ou privés. 


\title{
Les échelles d'équivalence complètes: une estimation intégrant les dimensions monétaire et temporelle des dépenses des ménages ${ }^{1}$
}

\author{
François Gardes ${ }^{2}$, Imen Sayadi ${ }^{3}$ Christophe Starzec ${ }^{4}$
}

\begin{abstract}
Résumé
L'appariement statistique d'une enquête de Budgets des Famille et d'une enquête de Budgets-Temps (enquêtes INSEE 2000) permet d'estimer, après valorisation du temps, les dépenses complètes des ménages, qui intègrent leur usage du temps disponible pour une production domestique à leurs dépenses monétaires. Le coût complet des enfants qui s'en déduit par les méthodes classiques d'estimation d'un système de demande s'avère supérieur au seul coût monétaire, ce que l'on observe également dans une moindre mesure pour le coût du second adulte. Les économies d'échelle semblent plus fortes donc dans la dimension temporelle que pour les dépenses monétaires. Le calcul des revenus complets que cet appariement permet d'opérer montre enfin que l'inégalité est moindre dans cette double dimension monétaire et temporelle que pour les seuls revenus monétaires.
\end{abstract}

\section{échelle d'équivalence, coût de l'enfant, allocation du temps, dépense complète, inégalité des revenus, pauvreté}

The full equivalence scales: an estimation integrating the monetary and time dimensions of households' expenditures

\begin{abstract}
The statistical match of a Family Budgets survey and Time Use survey (INSEE 2000) makes possible, once evaluated the cost of the time, the estimation of the full expenditure of household integrating the value of the domestic production and the monetary expenditure. The full cost of the child computed using the classic methods of demand systems estimation is higher than the child cost obtained by the monetary approach. To a lesser extent the same result is obtained for the cost of a second adult. The economies of scale seem to be stronger in the time use dimension than for monetary expenditure. Also, the inequality of the full incomes obtained by the matching is lower than those computed on monetary incomes only.
\end{abstract}

equivalence scale, child cost, time allocation, monetary expenditure, full expenditure, income inequalities, poverty

Classification JEL : C33, D1, D13, J22

\footnotetext{
${ }^{1}$ Nous remercions pour leurs commentaires et suggestions Stéphane Gauthier et les participants aux colloques ESPE (Chicago, 2007), JMA (Sousse, 2011).

${ }^{2}$ Paris School of Economics, Université Paris I Panthéon-Sorbonne,Centre d'Economie de la Sorbonne, 106-112 Bld. de l'Hôpital , 75013 Paris, courriel : gardes @univ-paris1.fr

${ }^{3}$ Institut Supérieur de Commerce et de Comptabilité de Bizerte (ISCCB)

Zarzouna Bizerte, courriel: isayadi@hotmail.fr

${ }^{4}$ Paris School of Economics, CNRS, Centre d'Economie de la Sorbonne, 106-112 Bld. de l'Hôpital , 75013

Paris, courriel : starzec @univ-paris1.fr
} 


\section{Introduction}

La méthode classique pour calculer des échelles d'équivalence, qui permettent de comparer les dépenses de ménages de tailles diverses, s'opère par l'estimation de systèmes de demande où les dépenses partielles et totale sont mesurées par unité de consommation grâce à ces échelles. Les problèmes liés aux choix de spécification du système de demande et de la forme des échelles s'ajoutent à une indétermination fondamentale des échelles (voir par exemple Lewbel [1989]) dues au fait que l'utilité du ménage change avec sa composition démographique (l'échelle de consommation se calculant en rapportant les dépenses des familles de structures démographiques différentes mais situées par leurs dépenses au même niveau d'utilité). Il est donc nécessaire de les estimer sous une hypothèse identifiante du niveau d'utilité (ou du niveau de vie) du ménage, par exemple en repérant l'utilité par la valeur du coefficient budgétaire des dépenses alimentaires (supposées d'après la loi d'Engel diminuer continument quand le niveau de vie du ménage augmente).

Une autre limite des échelles classiques tient au fait qu'elles n'intègrent pas les dépenses non monétaires (en particulier temporelles) faites pour les enfants ou les adultes supplémentaires. Ainsi le temps utilisé par les parents pour l'éducation et la prise en charge des enfants, qui se décide en substitution avec les usages alternatifs (travail marchand, loisir, autres productions domestiques), est-il complètement ignoré dans le calcul des échelles monétaires classiques, alors même qu'il implique des changements importants de la budgétisation des ressources monétaires du ménage. Apps [2000] suggère que le fait de se limiter à la seule dimension monétaire du coût de l'enfant revient à en ignorer la question essentielle ( the key issue »). On peut anticiper que le coût du temps dépensé auprès des enfants dans toutes les activités du ménage (pas seulement le caring : la présence d'enfant impacte les temps de transport, de courses alimentaires, etc...) peut dépasser le coût monétaire, et entraîner une croissance relative du coût complet avec le niveau de vie du ménage (au contraire du coût monétaire). On définira ce " coût complet » de l'enfant et plus généralement les «échelles d'équivalence complètes » comme l'expression de la totalité de ces dépenses monétaires et non-monétaires liées à la structure familiale (la dépense temporelle étant évaluée à partir de la mesure d'un coût d'opportunité du temps du ménage).

Nous proposons dans cet article une méthode originale pour estimer ces échelles complètes à partir d'un appariement des enquêtes françaises de Budgets des familles INSEE, BDF [2000] et de Budgets-Temps INSEE, BdT [1998]. On notera que l'agrégation des coûts monétaires et non-monétaires peut aider à définir le niveau de vie des ménages (cette identification est essentielle pour la comparaison de ménages équivalents de ce point de vue mais à structures familiales différentes, voir Pollack-Wales [1979] et Blundell-Lewbel $\left.[1991]^{5}\right)$. Par ailleurs, les modèles usuels du coût de l'enfant ne tiennent pas compte des effets de substitution entraînés par la modification du coût unitaire des diverses consommation selon leur degré de consommation publique - qui change avec la composition démographique du ménage (diminution par exemple du coût unitaire des transports privés avec la taille de la famille) : cet effet, repéré théoriquement par Barten [1964] («un litre de lait coûte relativement plus cher pour une famille nombreuse ») ne peut être estimé que si l'on dispose de prix différenciés entre les ménages, ce qui n'est pas commun. L'appariement entre des enquêtes de budgets monétaires ou temporels va permettre de construire des indicateurs de rareté utilisables comme des proxy de ces prix généralisés (voir Gardes [2013]). On remarquera enfin que l'appariement des deux enquêtes contiendra moins de dépenses nulles,

\footnotetext{
${ }^{5}$ Blundell et Lewbel [1991] ont montré qu'on ne pouvait identifier le coût de l'enfant à partir de statistiques transversales et que l'impossibilité de mesurer l'utilité du ménage permettait de dériver des données n'importe quel coût.
} 
ce qui évite le problème d'erreur de mesure classique sur enquête de consommation (une dépense nulle pendant la semaine d'enquête correspond-t-elle à une absence permanente de consommation ? $)^{6}$.

La première section présente les modèles utilisés et les hypothèses qui permettent d'identifier les échelles d'équivalence; la deuxième les deux enquêtes de l'INSEE et les méthodes d'appariement et de valorisation du temps des activités domestiques ; la troisième les estimations des coûts de l'enfant monétaire et complet et une méthode de mesure d'un revenu complet permettant un calcul des inégalités de revenu qui est comparé à l'inégalité des revenus monétaires des ménages.

\section{Section 1. L'estimation du coût de l'enfant et des échelles d'équivalence.}

\subsection{Spécification d'Engel de l'échelle monétaire classique}

On peut utiliser, conformément à la loi d'Engel, les changements de la part budgétaire de l'alimentation comme un indicateur du niveau de vie de ménage : la part alimentaire est supposée augmenter avec l'arrivée de l'enfant et ce changement correspond à une baisse du niveau de vie. L'estimation du coût de l'enfant consiste donc à régresser la part alimentaire sur le revenu (ou sur la dépense totale) ainsi que sur la taille et d'autres indicateurs de la structure démographique de la famille. Dans nos estimations, nous utiliserons le modèle Almost Ideal (AI Demand System) proposé par Deaton et Muellbauer [1980].

Ce système de demande est utilisé à la fois pour l'estimation sur données monétaires et pour celle en dépenses complètes (cela suppose que l'optimisation d'une fonction de coût Piglog vaut pour ces deux types de dépenses). On peut discuter cette hypothèse simplificatrice puisqu'une forme non linéaire commune aux spécifications monétaire et complète suppose une forme différente et compliquée de la demande de temps : on pourrait donc supposer alternativement que l'optimisation s'opère indépendamment par un coût Piglog sur les deux dimensions monétaires et temporelles, et que les paramètres de la fonction de demande complète se déduisent de ceux qu'on estime dans ces deux dimensions (voir Gardes, 2014). Il n’a pas été jugé utile de compliquer ainsi les spécifications pour cet article.

Pour un ménage $i$ la forme fonctionnelle de la part budgétaire alimentaire s'écrit :

$$
\mathrm{w}_{\mathrm{f}}=\alpha+\beta \ln x+\gamma^{*} \mathrm{f}(n)+\mathrm{Z} \zeta+\mathrm{u}
$$

avec $\mathrm{w}_{\mathrm{f}}$ la part budgétaire alimentaire, $\mathrm{x}$ le revenu total du ménage, $\mathrm{f}(n)=n$ ou $\ln (n)$ (ou une fonction du nombre d'enfant) et $\mathrm{Z}$ les diverses variables socio

-démographiques. L'échelle d'équivalence (ES) pour un couple avec un enfant par rapport à un couple sans enfants est obtenue par la formule suivante : ES $=\exp \left(-\gamma^{*} / \beta\right)$. On voit que selon cette formulation, l'alimentation est un bien nécessaire et que la part budgétaire d'alimentation augmente avec le nombre d'enfant. Cette méthode doit fournir une estimation du coût de l'enfant positif issue de l'estimateur de $\gamma^{*}$ positif et d'une valeur négative de $\beta$ (correspondant à une élasticité-revenu infra-unitaire des dépenses alimentaires).

L'identification de l'utilité avec la part budgétaire alimentaire est contestable en particulier dans les pays développés, où la part budgétaire de l'alimentaire est de l'ordre de

\footnotetext{
${ }^{6}$ L'appariement d'enquêtes a encore la vertu de permettre une mesure d'un revenu complet, intégrant la valeur du temps disponible pour la production domestique, qui constitue certainement un indicateur plus fidèle du bienêtre du ménage que son seul revenu monétaire. Enfin, l'information statistique est connu dans les budgets-temps au niveau des individus composant la famille, ce qui peut permettre de tester efficacement les modèles collectifs des choix familiaux.
} 
$15 \%$. De plus elle ne permet pas de calculer une échelle de consommation pour chaque type de bien comme la spécification suivante.

\subsection{Le modèle de Prais-Houthakker}

L'avantage principal du modèle Prais-Houthakker est permettre le calcul des échelles d'équivalence pour toutes les catégories de dépenses du budget global. L'échelle de la dépense totale (ou l'échelle de revenu) est alors obtenue comme une moyenne des échelles spécifiques pondérées par les coefficients budgétaires (une justification est donnée par Cramer [1969] ) .

Dans ce modèle les coefficients d'échelle sont calculés en estimant les fonctions de demande qui décrivent les changements de la consommation du ménage en fonction de sa structure démographique pour un niveau donné de bien être : il faut donc spécifier l'indicateur de bien-être utilisé :

$$
\frac{q_{i}}{m_{i}}=g_{i}\left(\frac{x}{m_{0}}\right)
$$

où $m_{0}$ est l'échelle de la dépense totale et $m_{i}$ les échelles spécifiques pour les bien $i$. Pour le ménage de référence (par exemple un adulte célibataire), toutes ces échelles sont fixées à 1.

L'échelle globale $m_{o}$ (applicable à la dépense totale ou au revenu du ménage) peut s'écrire comme une somme pondérée des échelles individuelles particulières à chaque bien; la pondération s'opère, selon les hypothèses faites, soit par les propensions moyennes à consommer calculées sur les consommations individuelles (c'est-à-dire corrigées par les échelles d'équivalence; c'est la spécification proposée par Cramer (1969) que l'on a reproduite en Annexe II), soit sur les consommations totales du ménage (la spécification proposée par Deaton-Muellbauer, 1980, p. 198, équation 2.7).

Notre spécification des fonctions de demandes est dérivée de la forme de Working-Leser (AIDS) de la courbe d'Engel. La dépense équivalente $e_{i}$ sur le bien $i$ s'écrit donc:

$$
\mathrm{e}_{\mathrm{i}} / \mathrm{m}_{\mathrm{i}}=\left[\mathrm{A}_{\mathrm{i}}\left(\mathrm{x} / \mathrm{m}_{0}\right) \ln \left(\mathrm{x} / \mathrm{m}_{0}\right)+\mathrm{B}_{\mathrm{i}}\left(\mathrm{x} / \mathrm{m}_{0}\right)\right]
$$

En divisant la dépense $e_{i}$ par $x$ et en multipliant par $m_{i}$ on obtient une formulation en termes de parts budgétaires. En ajoutant les vecteurs de variables socio démographiques $z$ et du terme d'erreur qui suit la loi normale, nous obtenons l'équation estimée suivante :

$$
\mathrm{w}_{\mathrm{i}}=\mathrm{m}_{\mathrm{i}} / \mathrm{m}_{0}\left[\mathrm{~A}_{\mathrm{i}} \log \left(\mathrm{x} / \mathrm{m}_{0}\right)+\mathrm{B}_{\mathrm{i}}\right]+\sum \delta_{\mathrm{s}} \mathrm{z}_{\mathrm{s}}+\varepsilon_{\mathrm{i}} .
$$

Pour tous les biens $i$ et pour la dépense totale nous avons respectivement les échelles :

$$
\mathrm{m}_{\mathrm{i}}=1+\alpha_{\mathrm{i}} * \mathrm{~N}_{\mathrm{c}}+\beta_{\mathrm{i}} *\left(\mathrm{~N}_{\mathrm{a}}-1\right), \mathrm{m}_{0}=1+\alpha_{0} * \mathrm{~N}_{\mathrm{c}}+\beta_{0} *\left(\mathrm{~N}_{\mathrm{a}}-1\right)
$$

où $\mathrm{N}_{c}$ est le nombre de personnes âgées de 18 ans et moins et $\mathrm{N}_{a}$ le nombre de personnes âgées de plus de 18 ans. L'hypothèse identifiante utilisée est que les dépenses d'alcool et de tabac sont nulles pour un enfant, ce qui implique que les dépenses d'alcool et de tabac ne changent pas si on compare les couples avec et sans enfants au même niveau de bien être. Cette hypothèse de Rothbarth assez discutée dans la littérature, du fait de la difficulté de repérer des biens assignables, c'est-à-dire uniquement utilisés par les adultes (vêtements...). Elle est utilisée ici pour l'identification des paramètres du modèle et non pour mesurer le 
niveau d'utilité atteint par un ménage. On notera que les approches d'Engel et de PraisHouthakker ne prennent pas en compte l'influence possible des prix relatifs sur les dépenses de familles ayant les structures démographiques différentes (c'est-à-dire le fait que le système de prix relatifs dépend, comme l'a indiqué Barten [1964], de la structure démographique du ménage et doit donc être pris en compte dans l'estimation). Ce problème est discuté dans Gardes et Starzec [2013].

\section{Section 2. L'appariement des données de budgets temps avec les données sur la consommation.}

\subsection{Modèle de l'allocation du temps}

La présence d'enfants dans le ménage entraîne des dépenses monétaires directes (et aussi des économies d'échelles) et une modification de l'allocation de son disponible par les adultes liée d'une part à des activités nouvelles pour les enfants («caring »), d'autre part à la modification du système de production du ménage (nouveau partage des taches, changement des temps de travail marchand des adultes, production domestique effectuée par les enfants...). Au lieu de mesurer sur les enquêtes le seul temps consacré explicitement aux enfants, comme le font la plupart des articles qui traitent du coût en temps des enfants (par exemple dans les articles de Bittman et Goodwin, 1998, ou de Bradbury, 2004), on s'intéresse dans cet article à tous les changements du budget-temps des adultes assignables à la présence d'enfants (même si ces temps d'activité domestique ne concernent pas directement les enfants : on modifie la technique de préparation du repas, on effectue des productions jointes à la garde des enfants...), donc issues de la comparaison des budgets-temps de famille comparables avec ou sans enfants.

Ceci est opéré dans le cadre du modèle d'allocation du temps de Becker (1965) qui affecte un temps unitaire à la production de chaque activité domestique (par exemple une heure et demi pour la préparation d'un repas) et définit un revenu complet et un temps complet en valorisant ces temps domestiques par un coût d'opportunité du temps, défini ici comme l'équivalent marchand du temps disponible (pour le ménage ou pour une activité à faible productivité). Les résultats obtenus encourent donc les critiques qui ont été faites à ce modèle,

Becker (1965) considère un ensemble de biens finaux dont les quantités $\mathrm{Z}_{\mathrm{i}}, \mathrm{i}=1 \mathrm{à} \mathrm{m}$, sont les déterminants de la fonction d'utilité directe du consommateur $u\left(Z_{1}, Z_{2}, \ldots Z_{m}\right)$. dans un but de simplification, Becker suppose que chaque activité i produit un seul bien final en quantité $\mathrm{Z}_{\mathrm{i}}$ avec deux facteurs pour chaque unité produite: un bien marchand unique ${ }^{7}$ en quantité xi et un temps unitaire $\tau_{i}$ par unité de bien marchand utilisé. Le temps total de production d'une unité de bien final sera donc $t_{i}=\tau_{i} x_{i}$. Les biens finaux sont donc produits selon des fonctions de production domestique: $Z_{i}=f_{i}\left(x_{i}, \tau_{i} ; W\right), \mathrm{W}$ représentant l'ensemble des autres facteurs de production identifiés aux caractéristiques socio-économiques du ménage. Le programme du consommateur s'écrit donc :

$\operatorname{Max} u\left(Z_{1}, Z_{2}, \ldots Z_{m}\right)$

Sous les contraintes: $Z_{i}=f_{i}\left(x_{i}, \tau_{i} ; W\right), \sum_{i} p_{i} x_{i}=y$ et $\sum_{i} \tau_{i} x_{i}+t_{w}=T$

$\mathrm{y}=\mathrm{wt}_{\mathrm{w}}+\mathrm{V}$ étant le revenu monétaire du consommateur sommant son revenue du travail et ses autres revenus,, $\mathrm{t}_{\mathrm{w}}$ le temps de travail marchand et $\mathrm{T}$ le temps disponible total pour le travail

\footnotetext{
${ }^{7}$ La multiplicité des biens marchands produisant un bien final s'exprime facilement matriciellement (voir AppsRees, 2010).
} 
marchand ou domestique sur la période envisagée. Les deux dernières contraintes permettent de definer le revenu complet (full income) $y^{f}$ comme le revenu monétaire maximal obtenu en consacrant tout son temps disponible $\mathrm{T}$ au travail marchand pour un taux de salaire net d'impôt w:

$$
y^{f}=w T+V=y+w\left(T-t_{w}\right)=y+w \sum_{i} \tau_{i} x_{i}
$$

La dépense totale pour la production d'une unité de bien final i s'écrit donc pour le consommateur $\mathrm{h}:\left(\mathrm{p}_{\mathrm{i}}+\omega_{\mathrm{h}} \tau_{\mathrm{ih}}\right) \mathrm{x}_{\mathrm{ih}}$. Elle dépend de son temps de travail domestique $t_{i h}=\tau_{i h} x_{i h}$ et de son coût d'opportunité du temps $\omega_{\mathrm{h}}$ qui est supposé ici pouvoir différer du taux de salaire marchand net.

\subsection{Procédure d'appariement}

Dans la méthode originale que nous proposons, notre objectif est d'obtenir, regroupés en un seul item, les dépenses monétaires et les dépenses en temps valorisées à partir des informations des enquêtes de Budgets de Famille INSEE BdF [2001] et de Budget de Temps INSEE BdT [1999]. Cet exercice relativement difficile et nouveau nécessite quelques hypothèses arbitraires concernant l'équivalence entre le temps passé sur diverses activités et les dépenses monétaires cf. Gronau, Hammermesh [2006] pour la discussion): nous supposons ici une parfaite substitution entre temps de travail domestique ou de loisir et temps de travail marchand; par ailleurs, les temps d'activité domestique ou marchande sont valorisés par un coût d'opportunité du temps supposé homogène au sein du ménage, et défini de manière exogène (voir Gardes, 2013, pour une endogénéisation de ce coût d'opportunité).

Nous définissons huit types d'activités compatibles avec les données disponibles à la fois dans l'enquête $\mathrm{BdF}$ et $\mathrm{BdT}$ : le temps de préparation des repas $(\mathrm{BdT})$ - la dépense alimentaire $(B d F)$; le temps de ménage et d'entretien de la maison $(\mathrm{BdT})$ - les dépenses de logement (incluant le loyer imputé) $(B D F)$; le temps d'entretien des vêtements (BdT) - les dépenses d'habillement $(\mathrm{BdF})$; Le temps consacré à l'éducation $(\mathrm{BdT})$ - les dépenses d'éducation $(B d F)$; le temps consacré aux de soins de santé $(\mathrm{BdT})$ - les dépense de santé $(B D F)$; le temps de loisirs (BdT) - les dépenses de loisirs $(B d F)$; le temps de transport (BdT) - les dépenses de transport $(B D F)$; les temps pour diverses activités (BdT) - les dépenses diverses $(B d F)$.

Dans cet article, toutes les activités hors travail marchand et sommeil sont supposées concourir également à la production domestique: les loisirs, le repos sont supposés nécessaires à une bonne productivité des tâches domestiques substituables à des services marchands, comme le temps consacré à la nourriture, à la prise en charge des enfants ou au nettoyage. Tout usage de temps est donc supposé concourir à la production domestique et au bien-être des ménages. Le transport n'a pas été affecté à ses différents usages (loisir, achats de nourriture, d'habillement...) en raison du manque de détail des nomenclatures et des productions jointes spécifiques à ces activités de transport (achats sur le trajet pour le travail marchand...), ce qui restreint le temps d'alimentation à domicile à la préparation des repas.

Deux méthodes sont utilisées pour l'appariement des enquêtes : la première consiste à diviser la population totale en cellules avec l'âge et l'éducation du chef de famille, et la localisation du ménage comme variables discriminantes. Cette méthode permet, par le regroupement des ménages en trente cellules comparables dans les deux enquêtes, de traiter convenablement les erreurs de mesure et le problème des dépenses nulles. Pour chaque cellule de l'enquête $\mathrm{BdF}$, nous ajoutons la moyenne des temps d'activités obtenus sur la cellule comparable de l'enquête BdT. La seconde méthode consiste à apparier individuellement les 
dépenses par régression: les diverses utilisation du temps pour toutes les activités sont estimées sur le BdT en fonction d'un certains nombre de caractéristiques des ménages. Les équations estimées sont alors utilisées pour prédire le temps passé à ces activités dans les ménages correspondant (i.e. ayant ces mêmes caractéristiques) de l'enquête BDF. Nous obtenons ainsi au niveau individuel du ménage de l'enquête BdF des informations à la fois sur l'emploi du temps et les dépenses monétaires.

\subsection{La valorisation du temps des activités domestiques}

Différentes méthodes de valorisation du temps peuvent être utilisées pour donner la valeur monétaire au temps passé aux activités domestiques. Notre première hypothèse est que cette valeur est égale au coût d'opportunité de l'emploi marchand : on suppose donc que le temps est parfaitement échangeable entre les activités marchandes et non marchandes. Dans cette approche, on attribue aux individus qui travaillent leur rémunération horaire dans l'emploi marchand net d'impôt, à chaque individu qui ne travaille pas son taux de salaire horaire espéré sur le marché du travail. Ce salaire marchand potentiel des absents du marché du travail est estimé séparément pour l'homme et la femme en utilisant la méthode $\mathrm{d}^{\prime} H_{e c k m a n}$ à deux étapes ${ }^{8}$. La seconde hypothèse consiste à calculer la valeur du travail non marchand en appliquant uniformément le taux de salaire égal au minimum légal (SMIC) in 2000. Nous supposons de plus que le coût d'opportunité du temps de travail domestique ne dépend ni de la nature de l'activité domestique ni de la période ou du jour où elle est effectuée. Le ménage est considéré comme une unité où la distribution des budgets monétaires et non marchand est une décision du couple.

Ces hypothèses, sans doute très simplificatrices, pourraient être affinées dans les travaux ultérieurs. On notera en particulier divers effets probables d'hétérogénéité individuelle qui jouent dans un sens divergent selon que le chef de famille a un capital humain élevé ou bas : ainsi par exemple, une contrainte subie par un conjoint peu éduqué sur le marché du travail (impossibilité de trouver un emploi) pourrait l'amener à effectuer un travail domestique peu rentable (au-delà du niveau optimal où sa productivité dépasse son coût marginal) ; la valeur monétaire de ce travail domestique surabondant (mesuré par un taux de salaire marchand d'opportunité) serait donc surévaluée, entraînant une surévaluation du revenu complet du ménage. Ceci conduirait donc à une surestimation du coût d'opportunité et du revenu complet pour les ménages à capital humain faible comparativement aux familles à capital humain supérieur, et donc à une sous-estimation de l'inégalité de revenu complet entre les ménages ${ }^{9}$.

La valorisation du temps de production domestique par le taux de salaire marchand espéré pour un individu qui ne travaille pas pourrait être supposée sous-estimer la valeur de ce temps domestique dans la mesure où l'individu a choisi de ne pas travailler en raison d'un salaire marchand inférieur à sa valorisation de son temps disponible. Néanmoins, les estimations du coût d'opportunité du temps qui ont été opérées sur ces données française (Gardes, Full price elasticities and the value of time, Document de travail du CES, 2014) sont de deux-tiers du taux de salaire marchand net, ce qui prouve que le choix de restreindre les activités

\footnotetext{
${ }^{8}$ Dans l'équation de participation, les variables explicatives comprennent le niveau d'éducation, l'âge, le type de ménage et le nombre d'enfant de moins de 5 ans. L'équation de salaire inclut l'éducation, l'âge et la catégorie socioprofessionnelle de l'individu. On a appliqué aux salaries espérés estimés le barème de l'impôt sur le revenu en tenant compte du nombre de jours travaillés et du nombre d'heures par jour. Cette méthode a été utilisée pour les données cellulées ainsi que pour les enquêtes appariées au niveau individuel. L'impôt sur le revenu est calculé pour chaque cellule avec les moyennes par cellule du revenu imposable et du nombre de parts.

${ }^{9}$ Des remarques de Stéphane Gauthier sont à l'origine de cette discussion.
} 
marchandes ne peut être entièrement expliqué par la faiblesse du salaire marchand espéré. La manière alternative de procéder (estimation du prix implicite du loisir) a été expérimentée dans l'article sus-mentionné mais pas intégré à l'article pour ne pas en compliquer la structure. Il est peu probable que la valorisation des temps domestique par un coût d'opportunité estimé change beaucoup l'estimation des échelles dans la mesure où les résultats qualitatifs relatifs à ces échelles ne changent pas selon qu'on emploie le salaire marchand réel ou estimé, ou le salaire minimum, pour valoriser ce temps domestique.

Dans le cas de la méthode d'appariement par cellulage, la valeur moyenne des activités non marchandes par cellule $(\omega)$ a été calculée comme la moyenne pondérée des taux de salaires pour l'homme et pour la femme ${ }^{10}: \omega=\mathrm{w}_{\mathrm{m}} * \operatorname{dom}_{\mathrm{m}}+\mathrm{w}_{\mathrm{f}}{ }^{*} \mathrm{dom}_{\mathrm{f}}$, avec $\mathrm{w}_{\mathrm{m}}$ : la rémunération horaire de l'homme dans la cellule et $\mathrm{w}_{\mathrm{f}}$ : la rémunération horaire de la femme dans la cellule, $\operatorname{dom}_{\mathrm{m}}$ le taux moyen de participation de l'homme dans les activités domestiques, $\operatorname{dom}_{\mathrm{f}}$ le taux moyen de participation de la femme dans les activités domestiques. En utilisant cette formule on peut calculer le coût moyen du temps pour chaque cellule et pour toutes les activités comme le produit du temps moyen et du coût d'opportunité horaire. ${ }^{11}$

Avec les dépenses complètes obtenues en additionnant toutes les dépenses monétaires $(\mathrm{BdF})$ et la valeur de la production issue de chacune des activités domestiques (BdT), on peut ainsi obtenir des échelles d'équivalence complètes en utilisant les méthodes traditionnelles d'estimation des échelles monétaires.

Les estimations supposent que la production domestique s'opère à partir des deux facteurs constitués par la dépense monétaire consacrée à une activité (produisant un bien final) et du temps consacré à cette activité. Cette substitution est complète, tant dans la définition du revenu complet que dans la spécification qui peut être définie pour les fonctions de production (par des fonctions de Cobb-Douglas par exemple dans l'article de Gardes, 2014). Cette substitution s'opère avec un même coût d'opportunité du temps dans la contrainte budgétaire complète (le choix de l'arbitrage entre les divers travaux domestiques et le travail marchand dépend donc du coût relatif unique de ces deux type de travail) se font donc, mais avec des paramétrages éventuellement locaux (spécifiques à chaque ménage) pour les fonctions de production domestique, ce qui donne un caractère de grande généralité à cette substituabilité productive. Il serait envisageable, mais avec un risque de faible robustesse des estimations, de considérer des coûts d'opportunité différenciés par activité domestique

\section{Section 3. Estimation des échelles d'équivalence complètes}

\subsection{Discussion des différentes méthodes d'estimation}

L'analyse de l'allocation du temps des ménages aux enfants est au moins aussi difficile à analyser que les dépenses monétaires, dans la mesure où l'usage de ce temps est très divers (éducation formelle ou informelle, jeux, lecture, toutes formes d'aide et d'accompagnement...) et où l'arrivée d'un enfant dans la famille distord de manière complexe les temps du ménage : non seulement de sa production domestique, mais également les temps de travail marchand et les temps physiologiques. L'analyse de ces choix ne peut se limiter aux seuls temps explicitement consacrés aux enfants, d'autant plus qu'ils comptent une forte proportion d'activités jointes. Une étude globale de l'allocation des ressources monétaires et temporelles selon les méthodes classiques d'analyse des choix monétaires s'impose donc.

\footnotetext{
${ }^{10}$ Le volume des activités non marchandes de l'homme actif est d'environ $2 \mathrm{~h} 36$ par jour. Pour une femme active, ce volume est de 3h53. Ainsi la répartition du volume des activités non marchandes est de 40\% pour l'homme et $60 \%$ pour la femme. Ces taux sont pratiquement identiques dans toutes les cellules.

${ }^{11}$ Calculé sur une base annuelle (l'enquête BdT donne des volumes journaliers).
} 
Les évaluations des coûts temporels des enfants sont beaucoup plus rares que celles des coûts monétaires. Parmi les plus intéressantes, C.Barnet-Versat et O.Ekert-Jaffé [2003] proposent un indicateur de bien-être basé sur le temps physiologique et de loisir qui permet de comparer les choix temporels des familles de taille différentes. La réduction de ce temps parental est interprété comme une perte d'utilité lié à la présence d'enfants, tant du fait des temps directement dédiés aux enfants que de l'augmentation de diverses tâches familiales. Pour ce faire, le temps parental est estimé sur le nombre d'enfants et leurs âges dans un système linéaire de dépenses en contrôlant la période, le jour de la semaine, la participation des adultes au marché du travail, leur statut social, leurs âges, la part du revenu de l'épouse dans le revenu familial, le statut marital, la localisation et la citoyenneté. L'estimation montre qu'un couple dépense en moyenne 50 minutes supplémentaires par jour pour un enfant unique agé de six à quatorze ans, 90 minutes pour un enfant de moins de six ans, sa dépense temporelle supplémentaire étant quasiment nulle dès que l'enfant dépasse quatorze ans. Bittman et Goodin [1998] estiment pour les dépenses en temps une échelle d'équivalence selon les mêmes méthodes que celles utilisées pour les dépenses monétaire et en déduisent le coût temporel de l'enfant. Bradbury [2004] adopte l'hypothèse identifiante de Rothbarth dont l'indicateur de bien-être est basé sur les biens exclusivement consommés par les adultes, ici le temps de loisir et d'activités personnelles. Le coût de l'enfant est alors défini comme la différence de revenu, entre un ménage sans et un ménage avec un enfant, permettant d'atteindre un même niveau de temps utilisé pour ces activités personnelles et de loisir. Le coût temporel de l'enfant ainsi estimé s'avère bien supérieur au coût monétaire, ce qu'il justifie par le fait que la présence d'un enfant a un plus fort impact sur le niveau de vie des parents (mesuré par leur temps d'activités personnelles) que celle d'un adulte supplémentaire.

Ces études ne tiennent pas compte de tous les changements des dépenses monétaires et temporels induits par la présence d'enfants, et ne peuvent permettre d'analyser les substitutions entre ces deux types de dépense.

\subsection{Résultat des estimations}

L'estimation des échelles complètes par le modèle de Prais-Houthakker est reportée dans la tableau 1, d'une part pour les données groupées en 38 cellules, d'autre part pour l'appariement individuel. Les deux calibrages du coût d'opportunité du temps, par le taux de salaire minimum ou par le taux de salaire net d'impôt moyen du ménage. Le deuxième tableau présente les estimations monétaires.

Les paramètres estimés dans les tableaux 1 et 2 représentent les coefficients des échelles de consommation. Par exemple le paramètre 0.39 signifie que le poids de la dépense alimentaire pour un enfant est de $39 \%$ de celle du premier adulte du ménage (homme ou femme). La dernière colonne de tous les tableaux représente une échelle dite « de revenu » ou «globale» avec le poids de toutes les dépenses pour un enfant par rapport au premier adulte. Dans la ligne correspondante à 0.39 ce poids est de 0.38 . Ce paramètre peut être aussi obtenu comme la moyenne pondérée (par les coefficients budgétaires) de tous les coefficients correspondants à des dépenses spécifiques (la première ligne du tableau sauf la dernière colonne). L'équation (5) définit ainsi l'échelle de consommation spécifique pour un bien $i$ $\left(m_{i}\right)$ et l'échelle «de revenu ou globale» $\left(m_{o}\right)$ en prenant en compte la structure démographique du ménage (enfant, adulte).

L'imprécision de la déclaration de revenus dans les enquêtes et plus généralement des erreurs de mesure liées à cette variable nous a amenés à l'instrumenter. Les variances des estimateurs dans les tableaux 1 à 3 ont été corrigées du fait de l'existence de régresseurs générés (les 
temps prédits) par une méthode de bootstrap. La correction est de l'ordre de 10 à $50 \%$ des écarts-type, ce qui ne modifie pas la significativité qualitative des paramètres estimés.

Tableau 1

Echelles d'équivalence complètes (modèle de Prais-Houthakker)

\begin{tabular}{|c|c|c|c|c|c|c|c|c|c|c|}
\hline \multicolumn{11}{|c|}{ Echantillon cellulé (38 cellules)* } \\
\hline \multicolumn{11}{|c|}{ Coût d'opportunité du temps : taux de salaire net } \\
\hline & Alimentation & Logement & Vêtement & Santé & Transport & Education & Loisirs & $\begin{array}{l}\text { Alcool, } \\
\text { tabac }\end{array}$ & Autres & $\begin{array}{c}\text { Echelle de } \\
\text { revenu }\end{array}$ \\
\hline Enfant & $\begin{array}{l}0.39 \\
(0.11)\end{array}$ & $\begin{array}{l}0.02 \\
(0.008) \\
\end{array}$ & $\begin{array}{l}0.45 \\
(0.12)\end{array}$ & $\begin{array}{l}0.32 \\
(0.04)\end{array}$ & $\begin{array}{l}0.58 \\
(0.13)\end{array}$ & $\begin{array}{l}1.24 \\
(0.34)\end{array}$ & $\begin{array}{l}0.26 \\
(0.06)\end{array}$ & $0^{12}$ & $\begin{array}{l}0.20 \\
(0.03) \\
\end{array}$ & $\begin{array}{l}\mathbf{0 . 3 8} \\
(0.03)\end{array}$ \\
\hline Adulte & $\begin{array}{l}0.87 \\
(0.23)\end{array}$ & $\begin{array}{l}0.19 \\
(0.006) \\
\end{array}$ & $\begin{array}{l}0.70 \\
(0.28)\end{array}$ & $\begin{array}{l}0.54 \\
(0.07)\end{array}$ & $\begin{array}{l}1.74 \\
(0.30)\end{array}$ & $\begin{array}{l}0.81 \\
(0.31) \\
\end{array}$ & $\begin{array}{l}0.60 \\
(0.27) \\
\end{array}$ & $\begin{array}{l}0.89 \\
(0.11) \\
\end{array}$ & $\begin{array}{l}0.59 \\
(0.15) \\
\end{array}$ & $\begin{array}{l}\mathbf{0 . 6 8} \\
(0.10) \\
\end{array}$ \\
\hline \multicolumn{11}{|c|}{ Coût d'opportunité du temps : SMIC } \\
\hline & Alimentation & Logement & Vêtement & Santé & Transport & Education & Loisirs & $\begin{array}{l}\text { Alcool, } \\
\text { tabac }\end{array}$ & Autres & $\begin{array}{l}\text { Echelle de } \\
\text { revenu }\end{array}$ \\
\hline Enfant & $\begin{array}{l}0.32 \\
(0.13)\end{array}$ & $\begin{array}{l}0.03 \\
(0.006)\end{array}$ & $\begin{array}{l}0.48 \\
(0.09)\end{array}$ & $\begin{array}{l}0.37 \\
(0.04)\end{array}$ & $\begin{array}{c}0.47 \\
(0.08)\end{array}$ & $\begin{array}{l}1.70 \\
(0.52)\end{array}$ & $\begin{array}{l}0.21 \\
(0.06)\end{array}$ & 0 & $\begin{array}{l}0.18 \\
(0.02)\end{array}$ & $\begin{array}{l}\mathbf{0 . 3 3} \\
(0.02)\end{array}$ \\
\hline Adulte & $\begin{array}{l}1.20 \\
(0.60) \\
\end{array}$ & $\begin{array}{l}0.20 \\
(0.005) \\
\end{array}$ & $\begin{array}{l}0.61 \\
(0.23) \\
\end{array}$ & $\begin{array}{l}0.50 \\
(0.06) \\
\end{array}$ & $\begin{array}{l}0.99 \\
(0.32) \\
\end{array}$ & $\begin{array}{l}1.04 \\
(0.54) \\
\end{array}$ & $\begin{array}{l}0.71 \\
(0.30) \\
\end{array}$ & $\begin{array}{l}0.73 \\
(0.14) \\
\end{array}$ & $\begin{array}{l}0.36 \\
(0.13) \\
\end{array}$ & $\begin{array}{l}\mathbf{0 . 6 4} \\
(0.08) \\
\end{array}$ \\
\hline \multicolumn{11}{|c|}{ Appariement individuel BDF et BDT } \\
\hline \multicolumn{11}{|c|}{ Coût d'opportunité du temps : taux de salaire net } \\
\hline & Alimentation & Logement & Vêtement & Santé & Transport & Education & Loisirs & $\begin{array}{c}\text { Alcool, } \\
\text { tabac }\end{array}$ & Autres & $\begin{array}{l}\text { Echelle de } \\
\text { revenu }\end{array}$ \\
\hline Enfant & $\begin{array}{l}0.66 \\
(0.04)\end{array}$ & $\begin{array}{l}0.40 \\
(0.03)\end{array}$ & $\begin{array}{l}1.41 \\
(0.08)\end{array}$ & $\begin{array}{l}0.95 \\
(0.09)\end{array}$ & $\begin{array}{l}0.88 \\
(0.05)\end{array}$ & $\begin{array}{l}1.24 \\
(0.34)\end{array}$ & $\begin{array}{l}0.40 \\
(0.04)\end{array}$ & 0 & $\begin{array}{l}0.14 \\
(0.03)\end{array}$ & $\begin{array}{l}\mathbf{0 . 5 8} \\
(0.04)\end{array}$ \\
\hline Adulte & $\begin{array}{l}1.10 \\
(0.04) \\
\end{array}$ & $\begin{array}{l}0.64 \\
(0.03) \\
\end{array}$ & $\begin{array}{l}1.35 \\
(0.06) \\
\end{array}$ & $\begin{array}{l}1.05 \\
(0.09) \\
\end{array}$ & $\begin{array}{l}0.84 \\
(0.04) \\
\end{array}$ & $\begin{array}{l}0.15 \\
(0.03) \\
\end{array}$ & $\begin{array}{l}0.75 \\
(0.04) \\
\end{array}$ & $\begin{array}{l}1.03 \\
(0.06) \\
\end{array}$ & $\begin{array}{l}0.82 \\
(0.04) \\
\end{array}$ & $\begin{array}{l}\mathbf{0 . 8 1} \\
(0.03) \\
\end{array}$ \\
\hline \multicolumn{11}{|c|}{$\begin{array}{l}\text { uêtes BDF et BDT appariées. Le revenu est instr } \\
\text { Coût d'opportunité du temps : SMIC }\end{array}$} \\
\hline & Alimentation & Logement & Vêtement & Santé & Transport & Education & Loisirs & $\begin{array}{c}\text { Alcool, } \\
\text { tabac }\end{array}$ & Autres & $\begin{array}{c}\text { Echelle de } \\
\text { revenu }\end{array}$ \\
\hline Enfant & $\begin{array}{l}0.72 \\
(0.05)\end{array}$ & $\begin{array}{l}0.46 \\
(0.04)\end{array}$ & $\begin{array}{l}1.44 \\
(0.12)\end{array}$ & $\begin{array}{l}1.80 \\
(0.20)\end{array}$ & $\begin{array}{l}0.57 \\
(0.05)\end{array}$ & $\begin{array}{l}0.99 \\
(0.05)\end{array}$ & $\begin{array}{l}0.41 \\
(0.05)\end{array}$ & 0 & $\begin{array}{l}0.35 \\
(0.05)\end{array}$ & $\begin{array}{l}\mathbf{0 . 5 5} \\
(0.05)\end{array}$ \\
\hline Adulte & $\begin{array}{l}1.18 \\
(0.06\end{array}$ & $\begin{array}{l}0.66 \\
(0.05)\end{array}$ & $\begin{array}{l}1.39 \\
(0.06)\end{array}$ & $\begin{array}{l}1.34 \\
(0.19)\end{array}$ & $\begin{array}{l}0.78 \\
(0.06)\end{array}$ & $\begin{array}{l}0.04 \\
(0.03)\end{array}$ & $\begin{array}{l}0.68 \\
(0.06)\end{array}$ & $\begin{array}{l}0.61 \\
(0.05)\end{array}$ & $\begin{array}{l}0.54 \\
(0.05)\end{array}$ & $\begin{array}{l}\mathbf{0 . 7 8} \\
(0.06)\end{array}$ \\
\hline
\end{tabular}

Source statistique: Enquêtes INSEE de BdF et de BdT appariées.

*Estimation sur 38 cellules définies par le niveau d'éducation, la localisation et les classes d'âge.

**Estimation sur 10273 observations des enquêtes BDF et BdT appariées. Le revenu est instrumenté par la dépense totale du ménage et les caractéristiques sociodémographiques de chef du ménage.

Tableau 2

Echelles d'équivalence monétaires classiques (modèle de Prais-Houthakker)

\footnotetext{
${ }^{12}$ Hypothèse identifiante: la dépense d'alcool et tabac est égale à zéro pour les enfants
} 


\begin{tabular}{|l|c|c|c|c|c|c|c|c|c|c|}
\hline & Alimentation & Logement & Vêtement & Santé & Transport & Education & Loisirs & $\begin{array}{c}\text { Alcool, } \\
\text { tabac }\end{array}$ & Autres & $\begin{array}{c}\text { Echelle } \\
\text { de revenu }\end{array}$ \\
\hline Enfant & $\begin{array}{l}0.53 \\
(0.04)\end{array}$ & $\begin{array}{c}0.14 \\
(0.02)\end{array}$ & $\begin{array}{c}0.42 \\
(0.06)\end{array}$ & $\begin{array}{c}0.56 \\
(0.09)\end{array}$ & $\begin{array}{c}0.11 \\
(0.03)\end{array}$ & $\begin{array}{c}2.14 \\
(0.92)\end{array}$ & $\begin{array}{c}0.15 \\
(0.03)\end{array}$ & 0 & $\begin{array}{c}0.24 \\
(0.04\end{array}$ & $\mathbf{0 . 2 1}$ \\
$(0.03)$
\end{tabular}

Source statistique: Enquêtes INSEE de BdF et de BdT appariées. Estimation sur 10273 observations de l'enquête BDF. Le revenu est instrument par la dépense totale du ménage et les caractéristiques sociodémographiques de chef du ménage.

Une première conclusion est que la méthode d'évaluation du coût d'opportunité du temps ne change pas fondamentalement les résultats (le coût de l'enfant est légèrement supérieur pour l'évaluation par le taux de salaire net, qui valorise plus la dépense monétaire.). En deuxième lieu, le coût de l'enfant est nettement plus élevé pour la dépense complète, qu'on le considère par rapport au coût du premier adulte (de 33 à $58 \%$ contre $21 \%$ pour les dépenses monétaires) ou par rapport au coût du deuxième adulte (coût relatif équivalent, de l'ordre de 50\%, pour les estimations sur données groupées ; coût relatif complet supérieur de $28 \%$ sur données individuelles). Notons également que le coût du deuxième adulte est toujours supérieur à celui de l'enfant (hors le cas des dépenses d'éducation dont les estimations sont imprécises) et que les coûts relatifs complets des enfants par rapport aux adultes sont nettement supérieurs aux coûts monétaires pour les dépenses alimentaires, de transport et de loisir, en particulier pour l'évaluation sur données individuelles.

L'estimation du modèle d'Engel (Tableau 3) montre également que le coût complet de l'enfant (probablement surévalué par l'hypothèse identifiante par la dépense alimentaire ; on notera aussi que l'estimation est plus sensible au mode d'évaluation du temps) est supérieur au coût monétaire, confirmant ainsi le résultat du modèle de Prais-Houthakker.

Le tableau 3 présente les résultats sous une forme légèrement différente. On compare un couple avec un enfant avec un couple sans enfant » (et non un enfant comparé à un adulte comme dans les tableaux 1 et 2) en utilisant uniquement l'échelle « de revenu ». La méthode d'estimation ne change pas, ce qui change c'est la référence (un couple et non le premier adulte).

Tableau 3

Echelles d'équivalence complètes et monétaires

\begin{tabular}{|c|c|c|}
\hline Couples avec enfants & Méthode Engel & $\begin{array}{l}\text { Méthode Prais- } \\
\text { Houthakker }\end{array}$ \\
\hline $\begin{array}{c}\text { Données cellulées BDF et BdT: } \\
\text { échelles complètes }\end{array}$ & $\begin{array}{c}1.43 \\
(0.02) \\
\end{array}$ & $\begin{array}{c}1.23 \\
(0.02) \\
\end{array}$ \\
\hline $\begin{array}{c}\text { Appariements individuels BDF et BdT: } \\
\text { échelles complètes } \\
\text { échelles monétaires }\end{array}$ & $\begin{array}{c}1.88 \\
(0.02) \\
1.18 \\
(0.01)\end{array}$ & $\begin{array}{c}1.32 \\
(0.02) \\
1.22 \\
(0.01)\end{array}$ \\
\hline
\end{tabular}

Source statistique: Enquêtes INSEE de BdF et de BdT appariées. Estimation sur 10273 observations des enquêtes BDF et BdT appariées. Le revenu est instrumenté par la dépense totale du ménage et les caractéristiques sociodémographiques de chef du ménage. Valorisation du temps par le taux de salaire.

Dans l'ensemble, ces résultats révèlent que le coût complet de l'enfant dépasse son coût monétaire. Selon ces estimations des coûts des enfants et des adultes, le rapport des dépenses d'un couple avec un enfant et d'un couple sans enfant est respectivement de 1,18 et 
1.22 pour les dépenses monétaires (selon la méthode d'estimation, par l'hypothèse identificatrice d'Engel ou le modèle de Prais-Houthakker), alors que ces rapports s'élèvent à 1,88 et 1,32 pour les dépenses complètes mesurées par un appariement individuel des deux enquêtes (1,43 et 1,23 pour un appariement par données cellulées).

Au total, le poids budgétaire complet de l'enfant intégrant les choix monétaires d'offre de travail marchand et de consommation et la production domestique des ménages, s'avère donc nettement supérieurs au seul coût monétaire. Ceci se vérifie à la fois pour le poids absolu de l'enfant dans le budget et pour son poids relatif au second adulte (ce dernier poids intégrant le partage des dépenses entre dépenses privatives, telle l'alimentation ou l'habillement, et dépenses publiques du ménage, tel le logement). Dans la mesure où une famille peut substituer son travail et sa consommation domestique aux équivalents marchands, on sousestime donc le coût des enfants par la prise en compte des seules dépenses monétaires. Pour une famille contrainte dans l'une des deux dimensions (par exemple une famille dont le temps de travail marchand des deux conjoints est très élevée et qui ne peut augmenter sa production domestique), l'obligation de compenser par des services marchands la prise en charge domestique des enfants doit également en augmenter fortement le coût monétaire. Enfin, dans le dernier cas d'une famille disposant d'une large possibilité d'extension de son temps de travail domestique, la substitution de travail domestique à des services marchands liés aux enfants lui permet de diminuer le cout complet des enfants, mais ce coût, évalué par nos estimations, reste supérieur au seul coût monétaire.

Enfin, on note également que le coût complet de l'enfant doit tendre à augmenter avec le revenu monétaire de la famille, dans la mesure où le coût d'opportunité du temps est positivement corrélé à ce niveau de revenu (comme le montre l'estimation du coût d'opportunité opérée sur ces mêmes données, voir Gardes [2014]). Ceci va à l'encontre des résultats habituels (souvent invoqués pour valider une diminution des allocations familiales aux familles riches) tendant à indiquer, dans la statistique purement monétaire, une diminution du coût relatif des enfants pour les familles riches.

\subsection{Inégalité des revenus monétaires ou des revenus complets?}

Si l'on suppose que les ménages ont la possibilité de substituer du travail domestique à l'achat de biens et services marchands - sous forme de dépense courante de consommation ou de dépense différée (c'est-à-dire d'épargne) -, il convient d'adjoindre à leur revenu monétaire (qui est la somme des deux consommations précédentes) l'équivalent monétaire de leur production domestique. Nous proposons donc de comparer l'inégalité des revenus complets des ménages français avec l'inégalité de leurs revenus monétaires en définissant le revenu complet comme la somme du revenu monétaire déclaré par le ménage et de l'équivalent monétaire de leur production domestique (valorisée par le salaire net moyen du ménage).

La seule connaissance des temps d'activité et du revenu par tranches dans l'enquête de Budgets-temps ne permet de mélanger ces deux formes de ressources en une seule que sous une hypothèse de valorisation du temps, qu'on ne peut obtenir que par l'appariement des enquêtes (ou, en l'absence d'une estimation du coût d'opportunité du temps, par son calibrage par le taux de salaire net moyen du ménage ou le taux de salaire minimum). Mais par ailleurs, ayant recollé les temps dans l'enquête de Budgets de famille, on peut utiliser dans le calcul du revenu complet le revenu du ménage connu en continu dans cette enquête. Il semble donc plus approprié d'effectuer ces calculs des inégalités en revenu complet sur l'enquête des Budgets de Famille. 
L'imputation d'une composante temporelle estimée par régression dans l'enquête budgétaire tend à sous-estimer la variance des temps, puisque la variance résiduelle de la régression sur les budgets-temps n'est pas contenue dans le temps affecté à l'enquête monétaire. Ceci pourrait être corrigé par des résidus simulés. La correction serait sans doute assez faible du fait de la grande partie de la variance expliquée par le régression des temps sur l'enquête de budgets-temps.

Par ailleurs, le revenu augmenté des ressources non monétaires doit être déflaté par l'application d'une échelle d'équivalence spécifique aux dépenses complètes, qui tendra donc à réduire le revenu complet par Unité de Consommation des ménages nombreux (donc à priori à revenu total élevé). Il faut noter enfin que les familles avec ou sans enfants, en fonction notamment de leur position dans leur cycle de vie, de leur taille et de leur capital humain, peuvent gérer différemment leur budget de temps entre les activités marchandes et non marchandes. Ces trois éléments modifieront la distribution des revenus que nous indiquerons ici par la seule dimension d'un indice d'inégalité des revenus.

On constate que ces trois effets contribuent à la diminution sensible de l'inégalité des revenus disponibles totaux des ménages, de 20,6\% pour l'indice de Gini et 41,2\% pour un indice de Theil. En second lieu, alors que la division du revenu par la taille du ménage ne change guère les indices d'inégalité, l'utilisation des échelles d'équivalence estimées sur les dépenses complètes réduit encore le niveau d'inégalité des revenus complets $(-22,4 \%$ par rapport à l'inégalité des revenus totaux pour l'indice de Gini, -45,2\% pour l'indice de Theil). Un semblable calcul de revenu complet par UC pour définir la situation de pauvreté par l'application du critère de pauvreté relative en deçà de $60 \%$ du revenu médian, réduit également le nombre de ménages pauvres (de $23 \%$ de la population pour le revenu monétaire total à $16 \%$ pour le revenu complet par unité de consommation).

Il apparait donc que la possibilité de substitution d'activités productives domestiques aux activités marchandes permet aux ménages pauvres d'améliorer leur positionnement relatif, ce qui réduit finalement les inégalités de revenu et sans doute modifie également leurs évolutions d'une période à l'autre.

\section{Conclusion}

Les échelles d'équivalences estimées à partir de l'appariement d'enquêtes de Budgets des Familles et de Budgets-Temps montrent que le coût complet des adultes supplémentaires (évalué par rapport au coût du premier adulte), et surtout le coût des enfants, sont nettement supérieurs aux niveaux indiqués par les échelles purement monétaires. Par ailleurs, le coût relatif des enfants tend à augmenter avec le revenu monétaire, ce qui contredit le constat usuel concernant les échelles monétaires. Ces résultats sont importants pour apprécier correctement les coûts familiaux dans le cas d'une possible substitution, au sein de la famille, du travail et des dépenses monétaires et temporelles, cas par exemple des familles aisées qui ont la possibilité d'augmenter leur offre de travail marchand et d'acquérir sur le marché des services destinés aux enfants. Ils impliquent une diminution sensible des niveaux d'inégalité des revenus quand on considère les revenus complets plutôt que les revenus monétaires.

Cette substitution et les coûts familiaux qui lui sont associés ont sans doute d'importante conséquence pour le partage intrafamilial du travail et des ressources, ce qui amène à proposer d'estimer les modèles collectifs de la famille avec ce type de données appariant les dimensions monétaires et temporels des choix familiaux (dont une particularité importante est qu'elles permettent de définir des coûts complets au niveau des observations individuelles - ici les ménages - et donc d'estimer les modèles avec des indices de prix 
informés au niveau individuel). La diminution des inégalités des revenus complets par rapport à celle des revenus monétaires est l'indice des modifications des ressources familiales et de l'allocation des ressources au sein de la famille liées à cette substitution entre travail domestique et travail marchand.

\section{Bibliographie}

BARNET-VERZAT C., EKERT-JAFFE O. [2003], «Le Coût du Temps Consacré aux Enfants », Communication aux 20èmes Journées de Microéconomie Appliquée.

BARTEN. A.P. [1964], «Family composition, prices and expenditure patterns, dans Economic Analysis for National Economic Planning, P. Hart, G. Mill et J. Whittaker, Eds., $16^{\text {th }}$ Meeting of the Colston Society, London, Butterworth.

BECKER G.S. [1965], «A Theory of the Allocation of Time », The Economic Journal, vol 75, p.493-517.

BITTMAN M., GOODIN R.E. [1998], « An Equivalence Scale for Time », Social Policy Research Centre, discussion paper $n^{\circ} 85$.

BLACKORBY, C., DONALSON, D [1991], « Adult Equivalence-Scales and Interpersonal Comparisons of Well-Being ", and Applied Welfare Economics, dans Interpersonal Comparisons and Distributive Justice, Elster J. and Romer J., Eds., Cambridge University Press.

BLUNDEL, R., LEWBEL, A. [1991], « The Information Content of Equivalence Scales », Journal of Econometrics.

BRADBURY B. [2004], "The price, Cost, Consumption and Value of Children, Social Policy Research Centre, discussion paper $n^{\circ} 132$.

CRAMER, J.S. [1969], Empirical Econometrics, Amsterdam: North Holland.

GARDES, F. [2014], «Full price elasticities and the opportunity cost for time », Cahiers CES Université Paris I, Panthéon Sorbonne.

GARDES, F., STARZEC, C. [2000], « Economies of Scale and Food Consumption: a Reappraisal of Deaton-Paxson Paradox », IARIW Conference Cracow 2000.

GARDES, F., STARZEC, C. [2013], «Individual prices and households' size: a restatement of equivalence scales using time and monetary expenditures combined », Cahiers CES Université Paris I, Panthéon Sorbonne.

GARDES F., DUNCAN G.J., GAUBERT P., STARZEC C. [2005], « Panel and PseudoPanel Estimation of Cross-Sectional and Time Series Elasticities of Food Consumption: The Case of U.S. and Polish Data », Journal of Business \& Economic Statistics , vol. 23, no. 2, 242-253.

GRONAU R, HAMERMESH D.S. [2006], « Time Vs. Goods: The Value of Measuring Household Production Technologies », Review of Income and Wealth, Series 52, $\mathrm{N}^{\circ} 1$, March 2006, 1-16.

INSEE, BDF [2001], voir l'Annexe : A. «Enquête de Budgets des Familles », INSEE, BDF 2000-2001.

INSEE, BtT [1999], voir l'Annexe : B. «Enquête de Budgets de Temps », INSEE, BdT, 1998-1999.

LEWBEL, A [1989], « Household Equivalence Scales and Welfare Comparisons », Journal of Public Economy », 39, 377-391

MUELLBAUER J. [1980], « The Estimation of the Prais-Houthakker Model of Equivalence Scales », Econometrica, vol.48, $\mathrm{n}^{\circ} 1,153-173$. 
PRAIS S.J., HOUTHAKKER H.S. [1955], « The Analysis of Family Budgets, with an Application to Two British Surveys Conducted in 1937-39 and their Detailed Results », Cambridge University Press. 


\section{Annexe I : Les sources de données}

\section{A. Enquête de Budgets des Familles, INSEE, BDF 2000-2001 13}

Le champ de l'enquête couvre les ménages habitant dans les logements ordinaires (à l'exclusion des institutions) en France métropolitaine et aussi parallèlement ceux habitant dans les départements d'Outre mer. La collecte de données est réalisée en plusieurs vagues (8) pendant un an sur deux années civiles à partir du printemps.

Le questionnaire est passé au cours de trois visites et fournit les caractéristiques sociodémographiques du ménage, ses dépenses régulières ou exceptionnelles, ses revenus pour l'année précédente et sa situation financière. Les périodes de référence sont variables selon le type de dépense (par exemple un an pour la résidence, les transports ou les biens durables, six mois pour les vacances ou les dépenses de santé, deux mois pour les vêtements et chaussures, un mois pour des services comme les soins aux enfants). Les carnets de dépenses sont remis au ménage à la première visite. Ils sont remplis par toutes les personnes âgées de 14 ans et plus pendant 14 jours (intervalle entre la première et la troisième visite). Comme les dépenses du ménage ne sont pas observées pendant un an il est nécessaire de prendre en compte des effets saisonniers dans la répartition de dépenses.

L'enquête BDF est longue et difficile pour les personnes enquêtées ce qui entraîne un risque de refus de participation élevé. Le taux de non-réponses peut ainsi atteindre $13 \%$ et le taux d'abandon en cours 12\%. Dans l'enquête 2000-2001 au total environ 10000 ménages ont répondu aux questionnaires et constituent la base de données utilisée.

Les résultats sont présentés en termes de dépenses et revenus annuels obtenus à partir des informations des carnets des dépenses et des questionnaires des vagues successives.

\section{B. Enquête de Budgets de Temps, INSEE, BdT, 1998-1999}

L'enquête Emploi du Temps de 1998-99 a été réalisée en tenant compte de l'harmonisation des statistiques au niveau européen. Elle avait pour but de faire progresser l'étude des comportements dans le domaine des activités de la vie quotidienne et des emplois du temps en quantifiant la durée des activités quotidiennes et la répartition dans une journée des quatre temps fondamentaux du quotidien : travail professionnel, travail domestique, temps libre et temps physiologique. L'enquête a été réalisée auprès d'un échantillon initial de 12000 logements tirés de façon uniforme. Le fichier exploitable contient 8186 ménages avec 15441 individus de plus de 15 ans interrogés (les budgets-temps sont en effet observés au niveau des individus). Les interviews ont été réalisées à l'aide de deux questionnaires distincts (ménage et individus) et s'appuient sur un carnet journalier d'activité où les personnes interrogées décrivent par tranches de dix minutes tout ce qu'elles ont fait au cours d'une de leur journée. Les caractéristiques de la journée de référence (journée normale de travail, jour de repos etc.) sont précisées. En plus du "carnet-journal", une grille de 11 activités permet d'obtenir plus d'informations sur la journée de travail (trajets, réunions, collaboration, contact avec le public, travail à la maison...).

La collecte a été faite en huit vagues de six semaines chacune étalée sur douze mois (du 16 février 1998 au 14 février 1999). L'interrogation s'organise en face à face en deux visites. Entre ces visites, tous les individus du ménage doivent remplir le même jour (fixé par l'enquêteur) un carnet d'activité où ils noteront leurs différentes occupations de dix minutes en

\footnotetext{
13 cf. Centre Maurice Halbwachs, http://www.cmh.ens.fr/greco/enquetes/XML/lil-0169.xml

14 cf. Centre Maurice Halbwachs, http://www.cmh.ens.fr/greco/enquetes/XML/lil-0169.xml
} 
dix minutes. Les carnets sont ramassés lors de la seconde visite. La durée de l'interrogation est de 70 minutes pour la première visite et de 20 minutes pour la seconde.

\section{Annexe II : Calcul de l'échelle d'équivalence globale}

Cramer (1969) écrit la courbe d'Engel par unité de consommation : $\frac{C_{j}}{F_{j}}=f_{j}\left(\frac{x}{F}\right)$ pour une consommation $C_{j}$, une échelle spécifique $F_{j}=\sum \lambda_{l_{j}} K_{l}$ fonction du nombre d'individus de type 1 dans le ménage $K_{l}$ et des poids $\lambda_{l_{j}}$ de ce type, $\mathrm{X}$ et $\mathrm{F}$ étant la dépense totale et la taille du ménage. On voit facilement que $\sum \frac{\partial C_{j}}{\partial x}=1$ et $\frac{\partial C_{j}}{\partial x}=\frac{F_{j}}{F} f_{j}^{\prime}$, soit $F=\Sigma F_{j} f_{j}$. La différenciation de $C_{j}=F_{j} f_{j}\left(\frac{X}{F}\right)$ par rapport à $\mathrm{K}_{1}$ fournit $: \frac{\partial C_{j}}{\partial K_{\mathrm{I}}}=\lambda_{l_{j}} f_{j}-\Lambda_{l} \frac{X}{F^{2}} F_{j} f_{j}^{\prime}$ en fonction de l'échelle globale $\Lambda_{l}$. On obtient donc finalement :

$$
\Lambda_{l} \frac{X}{F^{2}} \sum F_{j} f_{j}^{\prime}=\sum \lambda_{l_{j}} f_{j} \text { soit: } \Lambda_{l}=\frac{F}{X} \sum \lambda_{l_{j}} f_{j}=\sum \frac{\left({ }^{c j} / F_{j}\right)}{\left({ }^{X} / F\right)} \lambda_{l_{j}}
$$

